\title{
AN ENCODING TECHNIQUE BASED ON WORD IMPORTANCE FOR THE CLUSTERING OF WEB DOCUMENTS
}

\author{
John Zakos and Brijesh Verma \\ School of Information Technology \\ Griffith University, Gold Coast Campus \\ PMB 50 Gold Coast Mail Centre \\ QLD 9.726, Australia \\ E-mail: \{j.zakos, b.verma\}@gu.edu.au
}

\begin{abstract}
In this paper we present a word encoding and clustering technique that groups web documents based on the importance of the words that appear in the documents. We use a two level self-organizing map architecture to generate clusters of words and documents. We propose that by capturing word importance information of words, similar documents can be then clustered to assist in web document retrieval. A web document retrieval system is presented to demonstrate how this approach could be integrated into web search.
\end{abstract}

\section{INTRODUCTION}

It was recently reported [1] that the WWW was composed of approximately 800 million web pages spread over 3 million servers that amounted to about 6 terabytes of text data. With so much information on the Internet, there is a need for effective and useable systems to retrieve relevant web pages. Furthermore, there is a need for tools and frameworks for the searching [2-6], browsing [7-8], visualization [9-10], clustering [11] and categorizing [1213] of Internet information.

Document clustering [14] is an area within information retrieval that attempts to group documents based on a similarity measure. Clustering is different from categorization and classification in that there are no predefined classes specified for clustering. A clustering technique attempts to create classes of groups documents that are similar in some way. Various clustering approaches have been researched [15].

One method of clustering documents is through the use of self-organizing maps [16-17]. The most comprehensive and prominent system that clusters web documents based on self-organizing maps for retrieval and visualization is WEBSOM [11][18-19]. It uses a two level Kohonen's self-organizing map approach to group words and documents of similar contextual similarity. The first map, the word category map, attempts to group similar words based on their encoded context using a 270 dimensional feature vector that represents the contextual nature of a word.

To encode a word's context, each word is first assigned a random vector of 90 dimensions. Then all the words that occur before and after the considered word are collected and averaged and combined with the considered word to form the final 270 dimensional feature vector. All unique words are used to generate the word category map that is then used for document encoding. The document encoding for the second map, the document map, results in the generation of document histograms whose dimensions are based on the number of clusters from the word category map. Every word from every document is mapped to the corresponding dimension in the document histogram and then that dimension is incremented. The main advantage of this approach is that an entire document is represented as a histogram of only 315 dimensions. This document histogram is a "blurred" generalized representation of the document.

This paper focuses on an alternative word encoding technique to cluster web documents using selforganizing maps. It also describes how the technique will be integrated into current search engine technology to produce clusters of search results to improve a users search experience. The motivation behind this research is the fact that current search engines answer user queries with many thousands of results, most of which are irrelevant to the initial query and presented in an unorganized fashion. Thus, an effective and intuitive way of presenting search results in clusters to allow users to filter through and find relevant results quicker is needed. 


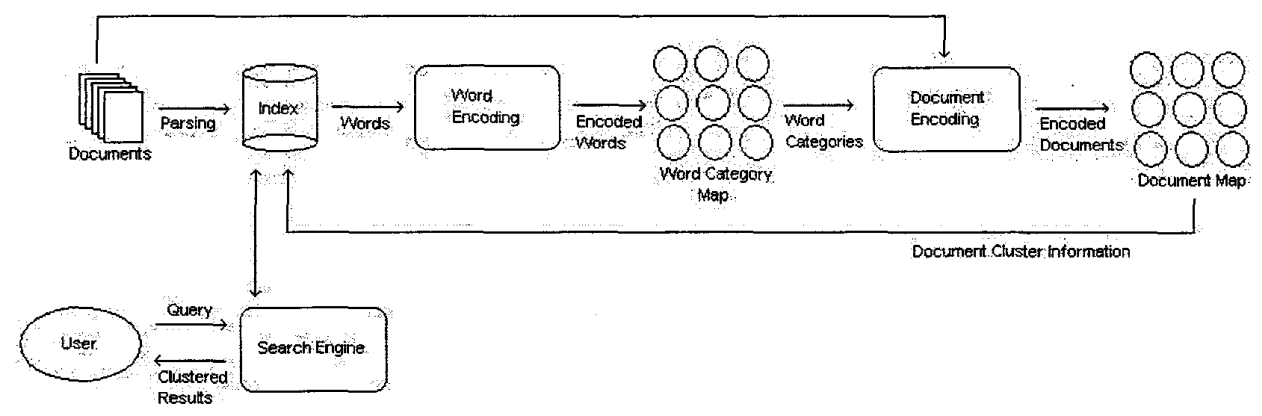

Figure 1. Architecture of Proposed Web Document Retrieval System

This paper is organized in 5 sections. The next section describes the proposed technique based on a two level self-organizing map approach. Section 3 presents the results of a preliminary experiment. Section 4 presents an analysis and discussion of results. In section 5 we conclude and outline the future direction for research.

\section{PROPOSED TECHNIQUE}

The proposed technique is based on a two level selforganizing map (SOM) approach. The first SOM, the word category map, groups words and is then used to generate document histograms that are passed as input feature vectors to the second SOM, the document map. The resultant document map is a $2 \mathrm{D}$ clustered organization of documents.

The main idea behind our approach is to end up with clusters of documents grouped by importance. We propose that the importance of a word gives a good indication of the type of document that word appears in. We also propose that words of similar importance tend to make up similar types of documents. We consider documents to be similar if they have the same type of information content (see Section 3). By capturing word importance information in the initial stages of our technique, documents can then be clustered according to how much information content they possess. Thus, importance can be interpreted as the amount of information content a particular document contributes to a particular theme.

Once clustering has been performed, document clusters can be analyzed and information can be extracted by the system to be used for the ranking and presentation of grouped search results. The overall architecture of the proposed web document retrieval system is depicted in Figure 1.

\subsection{Generating the Word Category Map (WCM)}

The WCM is a self-organizing map that attempts to cluster words based on their importance. A word is considered an important word for a document the more frequently it appears within that document and the less frequently it appears in other documents in the document collection. The importance of a word can be captured and encoded by the term frequency inverse document frequency [20] calculation:

$$
w_{i j}=t f_{i j} \times \log _{2} \frac{N}{n}
$$

where

$i \quad$ is an index to a term in a document.

$j$ is an index to a document in the document collection.

$w_{i j}$ is the weight (importance) of term $i$ in document $j$.

$t f_{i j}$ is the frequency of term $i$ in document $j$.

$N$ is the number of documents in the collection.

$n$ is the number of documents in the document collection where term $i$ occurs at least once.

For the WCM, the overall importance of a word is represented by a 3 dimensional feature vector. The first feature is an average of the importance of the words that occur before the considered word. The second feature is the averaged importance of the considered word. The third feature is an average of the importance of the words that occur after the considered word:

$$
\bar{w}=\left[\bar{w}_{\text {before }}, \bar{w}_{\text {considered }}, \bar{w}_{\text {after }}\right]
$$


Before a feature vector can be generated for a word, each term weight must first be calculated for every single word in every single document using the term frequency inverse document frequency calculation (1). Next, the average weight for each unique term $i$ is calculated:

$$
\overline{w_{i}}=\frac{\sum_{j=1}^{n} w_{i j}}{n}
$$

where

$$
\begin{aligned}
& \bar{w}_{i} \text { is the averaged weight for term } i \text { across the } \\
& \text { document collection. } \\
& \text { Refer to equation } 1 \text { for descriptions of } j \text { and } n \text {. }
\end{aligned}
$$

Once the average weights are calculated for each word in the collection, each corresponding feature vector is then created. For example, for the word $d o g$, each word that occurs before $d o g$ throughout the entire document collection is recorded and the average weight is computed. The same is done for every word after dog. These averaged before and after weights are then combined with the weight for $\operatorname{dog}$ and the 3 dimensional feature vector is now generated. The resulting feature vector for the word dog may look something like this:

$$
w_{d o g}=[0.345,0.555,0.976]
$$

With feature vectors created for every word in the document collection, the WCM can now be trained and tested. After the testing phase we have clusters of words grouped according to their importance. This is depicted in Figure 2.

\subsection{Generating the Document Map (DM)}

The trained and tested WCM is used during the document encoding to generate document histograms for each document in the collection. Each cluster (node) from the WCM is mapped to a single dimension in the document histogram. From the WCM depicted in Figure 2, the corresponding document histograms would be of 9 dimensions. To generate a document histogram for a particular document, consider each word in the document and increment by one the histogram dimension to which that word belongs. The resultant document histogram is then used as the input feature vector for the document map. The resultant trained and tested DM will contain clusters of documents based on a similarity of importance.

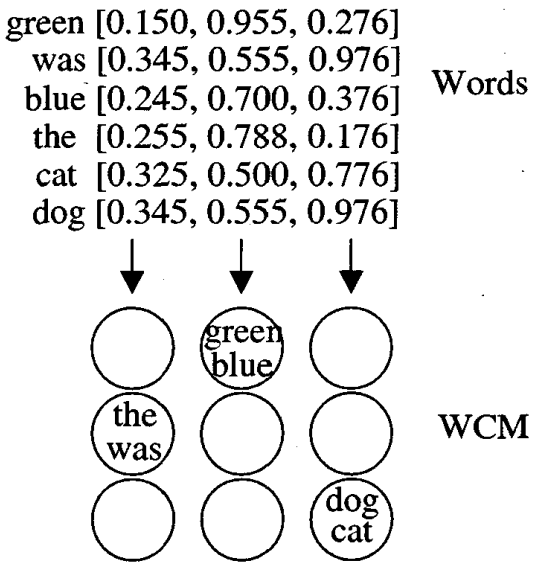

Figure 2. Word Category Map: Words of Similar Importance Cluster to Same Node

\subsection{Search Engine Integration}

We intend to integrate the proposed clustering technique into current search engine technology by making simple modifications to the index. After the DM is generated we know which documents belong to which clusters. This information is then stored back into the index. When a keyword search is performed and the search on the index returns matched documents, the results will be presented in a clustered manner. This will allow users to view clusters of results rather than just a flat long list of thousands on search results. The main unanswered questions that are outside the scope of this paper are

1. How is a cluster of documents best represented to a user?

2. How does the user best interact with the clusters and results?

One of the most appealing features of this approach is that since documents have been grouped by a similarity measure based on importance, search results can be ranked using this cluster information. While most search engines base their ranking on word occurrence and positioning, our approach will rank documents based on document and cluster importance that has been initially captured through the word encoding and mapping stage.

\section{EXPERIMENTAL RESULTS}

We have run a preliminary experiment using the word encoding technique outlined above. 68 web pages that mentioned the word "spiders" were used as the base document collection. These documents were obtained by crawling the internet. The documents varied in the 
information content they contained about "spiders". Each document was labeled with one of the categories from Table 1 depending on how much information content it had about "spiders". The information content of document reflects how important a document is to a particular theme. The importance of a document is measured by how much relevant information it has about "spiders". 18 documents had a label of "no relevant information content", 15 had a label of "some relevant information content" and the remaining 45 had a label of "all relevant information content".

In the 68 documents there were 10470 unique words. Each word was encoded and its corresponding feature vector was constructed and used to train and test the word category map. The word category map had a topology of $20 \times 20$ nodes and was trained for 100000 iterations (i.e. each words was inputted approximately 10 times). After the word category map was trained and tested on the same words, it was used to encode the document histograms for the document map. Each of the 68 documents was used for training and testing. The document map had a topology of $3 \times 3$ nodes and was trained for 680 iterations (i.e. each document histograms was inputted 10 times).

Table 1. Information Content (IC) Categories

\begin{tabular}{|l|l|}
\hline $\begin{array}{l}\text { No Relevant } \\
\text { Information } \\
\text { Content } \\
\text { (NRIC) }\end{array}$ & $\begin{array}{l}\text { The document contains the word } \\
\text { "spiders", but the document } \\
\text { information content is not about } \\
\text { spiders. }\end{array}$ \\
\hline $\begin{array}{l}\text { Some Relevant } \\
\text { Information } \\
\text { Content } \\
\text { (SRIC) }\end{array}$ & $\begin{array}{l}\text { The document information } \\
\text { content contains some section/s } \\
\text { about "spiders", but also contains } \\
\text { other sections about other } \\
\text { subjects. }\end{array}$ \\
\hline $\begin{array}{l}\text { All Relevant } \\
\text { Information } \\
\text { Content } \\
\text { (ARIC) }\end{array}$ & $\begin{array}{l}\text { The document information } \\
\text { content is all about "spiders" and } \\
\text { refers to no other subjects. }\end{array}$ \\
\hline
\end{tabular}

Table 2 outlines the classification results of the document technique clustering across all nodes in the DM. The document clustering performed well with 6 of the 9 nodes achieving a classification accuracy greater or equal to $70 \%$. Nodes 1,2 and 4 achieved an accuracy of $100 \%$. Nodes 5,6 and 8 had poorer classification rates of $66.6 \%$, $62.5 \%$ and $57.1 \%$ respectively.

\section{DISCUSSION OF RESULTS}

The resultant labeled DM is shown in Figure 3. Each node is labeled with one of the information content categories. We perform simple labeling by giving a node the label that corresponds to the information content category of the

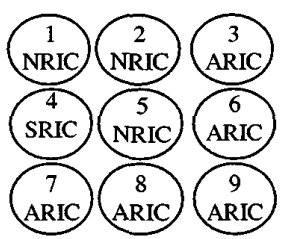

Figure 3. The labeled DM

Table 2. Document Classification Rates Per IC Category

\begin{tabular}{|c|c|c|c|}
\hline Node & \% NRIC & \% SRIC & \% ARIC \\
\hline $\mathbf{1}$ & $\mathbf{1 0 0 . 0}[\mathbf{4 / 4}]$ & $0.0[0 / 4]$ & $0.0[0 / 4]$ \\
\hline $\mathbf{2}$ & $\mathbf{1 0 0 . 0}[\mathbf{1 / 1}]$ & $0.0[0 / 1]$ & $0.0[0 / 1]$ \\
\hline $\mathbf{3}$ & $11.1[1 / 9]$ & $0.0[0 / 9]$ & $\mathbf{8 8 . 8}[\mathbf{8 / 9}]$ \\
\hline $\mathbf{4}$ & $0.0[0 / 5]$ & $\mathbf{1 0 0 . 0}[\mathbf{5} / 5]$ & $0.0[0 / 5]$ \\
\hline $\mathbf{5}$ & $\mathbf{6 6 . 6}[\mathbf{2 / 3}]$ & $0.0[0 / 3]$ & $33.3[1 / 3]$ \\
\hline $\mathbf{6}$ & $25.0[2 / 8]$ & $12.50[1 / 8]$ & $\mathbf{6 2 . 5}[\mathbf{5 / 8}]$ \\
\hline $\mathbf{7}$ & $0.0[0 / 8]$ & $28.5[2 / 7]$ & $\mathbf{7 1 . 4}[\mathbf{5} / 7]$ \\
\hline $\mathbf{8}$ & $23.8[5 / 21]$ & $23.8[5 / 21]$ & $\mathbf{5 7 . 1}[\mathbf{1 1} / 21]$ \\
\hline $\mathbf{9}$ & $0.0[0 / 10]$ & $30.0[3 / 10]$ & $\mathbf{7 0 . 0}[\mathbf{7 / 1 0}]$ \\
\hline
\end{tabular}

most number documents that were assigned to that node. For example, if a particular node was assigned 5 documents from the NRIC category and 2 documents from the SRIC category, then that node would be labeled with NRIC. Node 1 (top left) was labeled with NRIC as it was assigned 4 documents, all from the NRIC category. Node 3 (top right) was labeled with ARIC because it was assigned 9 documents from the ARIC category and only 1 document from the NRIC category.

Overall, these results displayed in Table 2 give us a good indication about the potential of this approach and the usefulness of the document cluster information created. Lets say a user was to use the proposed system to search for "spiders". Standard keyword matching search would find $X$ number of documents containing the word "spiders" and then these documents would be presented to the user as clusters of documents. Each cluster would be dominated by documents containing a similar type of information content. So if the user wanted only to read documents that were "all about spiders", then the appropriate documents can quickly be located and identified by the user through the clusters. Alternatively, the user may only want to read documents that mention the word "spiders" but are not about spiders. These documents could also be located quickly through the clusters.

\section{CONCLUSION}

We have proposed a word encoding and clustering technique to capture the importance of words across a document collection. This in turn allows us to cluster documents based on word importance similarity across 
documents. The information deduced from document clusters could be integrated into current search engine technology. Our initial experiment has achieved some promising results.

By capturing word importance, we have shown that similar types of documents can be clustered together according to their information content. Consequently, this shows that words of similar importance tend to occur in documents with similar levels of information content.

\section{REFERENCES}

[1] S. Lawrence and C. Lee Giles, "Accessibility of information on the web," Nature, vol. 400, no. 6740, pp. 107-109, 1999.

[2] S. Brin and L. Page, "The Anatomy of a Large-Scale Hypertextual Web Search Engine," Computer Networks, vol. 30, no. 1-7, pp. 107-117, 1998.

[3] A. Howe and D. Dreilinger, "SavvySearch: A MetaSearch Engine that Learns which Search Engines to Query." in AI Magazine, vol. 18, no. 2, pp. 19-25, 1997.

[4] Altavista, http://www.altavista.com.

[5] N. Zacharis and T. Panayiotopoulos, "Web Search using a Genetic Algorithm," IEEE Internet Computing, vol. 5, no. 2, pp.18-26, 2001.

[6] K. Bharat, "SearchPad: Explicit Capture of Search Context to Support Web Search," Computer Networks, vol. 33, no. 1-6, pp. 493-501, 2000.

[7] H. Lieberman. "Letzia: An Agent that Assists Web Browsing," in 1995 International Joint Conference on Artificial Intelligence, Montreal, Canada, pp. 924929, 1995.

[8] M. Pazzani, J.Muramatsu and D. Billsus, "Syskill \& Webert: Identifying Interesting Web Sites," in Proceedings of the National Conference on Artificial Intelligence (AAAI), Portland, USA, pp. 54-61, 1996.

[9] A. Nurnberger and M. Detyniecki, "Visualizing Changes in Data Collections Using Growing SelfOrganizing Maps," 2002 IEEE World Congress on Computational Intelligence, Hawaii, USA, 2002.

[10] A. Klose, A. Nurnberger, R. Kruse, G. Hartmann and M. Richards, "Interactive Text Retrieval Based on Document Similarities," Phys. Chem. Earth, vol. 25, pp. $649-654,2000$.
[11] T. Honkela, S. Kaski, K. Lagus and T. Kohonen, "WEBSOM - Self-Organizing Maps of Document Collections," in Proceedings of WSOM'97 (Workshop on Self-Organizing Maps), Espoo, Finland, pp. 310-315, 1997.

[12] J. Tin-Yau Kwok, "Automated Text Categorization using Support Vector Machine," in Proceedings of the International Conference on Neural Information Processing (ICONIP), Kitakyushu, Japan, pp. 347$351,1999$.

[13] T. Joachims, "Text Categorization with Support Vector Machines: Learning with Many Relevant Features," in Proceedings of the 10th European Conference on Machine Learning (ECML), Chemnitz, Germany, pp. 137-142, 1998.

[14] G. Kowalski, "Information Retrieval Systems: Theory and Implementation," Kluwer Academic Publishers, Norwell, 1997.

[15] A. Leouski and W. Croft, "An Evaluation of Techniques for Clustering Search Results," Technical Report IR-76, Department of Computer Science, University of Massachusetts, Amherst, 1996.

[16] X. Lin, D. Soergel and G. Marchioni, "A Selforganising Semantic Map for Information Retrieval," in Proceedings of the Fourteenth Annual International ACM/SIGIR Conference on Research and Development in Information Retrieval, New York, USA, pp. 262-269, 1991.

[17] T. Kohonen, "Exploration of Very Large Databases by Self-Organising Maps," in Proceedings of the IEEE International Conference on Neural Networks, Houston, USA, vol. 1, pp. 1-6, 1997.

[18] T. Honkela, "Self-Organizing Maps in Natural Language Processing," Ph.D. Thesis, Helsinki University of Technology, Neural Networks Research Center, Espoo, Finland, 1997.

[19] S. Kaski, K. Lagus, T. Honkela and T. Kohonen, "Statistical Aspects of the WEBSOM System in Organizing Document Collections," Computing Science and Statistics, vol. 29, pp. 281-290, 1998.

[20] R. Baeza-Yates and B. Ribeiro-Neto, "Modern Information Retrieval," Addison Wesley, New York, 1999. 